Bull. Korean Math. Soc. 50 (2013), No. 5, pp. 1587-1598

http://dx.doi.org/10.4134/BKMS.2013.50.5.1587

\title{
HARNACK INEQUALITY FOR A NONLINEAR PARABOLIC EQUATION UNDER GEOMETRIC FLOW
}

\author{
LIANG ZHAO
}

\begin{abstract}
In this paper, we obtain some gradient estimates for positive solutions to the following nonlinear parabolic equation

$$
\frac{\partial u}{\partial t}=\Delta u-b(x, t) u^{\sigma}
$$

under general geometric flow on complete noncompact manifolds, where $0<\sigma<1$ is a real constant and $b(x, t)$ is a function which is $C^{2}$ in the $x$-variable and $C^{1}$ in the $t$-variable. As an application, we get an interesting Harnack inequality.
\end{abstract}

\section{Introduction}

In this paper, we shall study the following nonlinear parabolic equation

$$
\frac{\partial u}{\partial t}=\Delta u-b(x, t) u^{\sigma}
$$

under general geometric flow on complete noncompact manifolds $M$, where $0<\sigma<1$ is a real constant and $b(x, t)$ is a function which is $C^{2}$ in the $x$-variable and $C^{1}$ in the $t$-variable.

Gradient estimates have played an important role in the study of PDE, especially the Laplace equation and the heat equation. Zhang and Ma [9] considered gradient estimates for positive solutions to the following nonlinear equation

$$
\triangle_{f} u+c u^{-\alpha}=0, \alpha>0
$$

on complete noncompact manifolds, where $\triangle_{f}=\Delta-\nabla f \cdot \nabla$. When $\mathrm{N}$ is finite and the N-Bakry-Émery Ricci tensor is bounded from below, the authors [9] got the following result.

Theorem 1.1 (Zhang and Ma [9]). Let $(M, g)$ be a complete noncompact $n$ dimensional Riemannian manifold with $N$-Bakry-Émery Ricci tensor bounded from below by the constant $-K=:-K(2 R)$, where $R>0$ and $K(2 R)>0$ in

Received August 27, 2012; Revised March 18, 2013.

2010 Mathematics Subject Classification. 53C44.

Key words and phrases. parabolic equation, positive solutions, geometric flow, Harnack inequality. 
the metric ball $B_{p}(2 R)$ around $p \in M$. Let $u$ be a positive solution of (1.2). Then

(1) if $c>0$, we have

$$
\begin{aligned}
\frac{|\nabla u|^{2}}{u^{2}}+c u^{-(\alpha+1)} \leq & \frac{(N+n)(N+n+2) c_{1}^{2}}{R^{2}}+\frac{(N+n)\left[(N+n-1) c_{1}+c_{2}\right]}{R^{2}} \\
& +\frac{(N+n) \sqrt{(N+n) K} c_{1}}{R}+2(N+n) K .
\end{aligned}
$$

(2) if $c<0$, we have

$$
\begin{aligned}
& \frac{|\nabla u|^{2}}{u^{2}}+c u^{-(\alpha+1)} \\
\leq & (A+\sqrt{A})|c|\left(\inf _{B_{p}(2 R)} u\right)^{-\alpha-1}+\frac{(N+n)\left[(N+n-1) c_{1}+c_{2}\right]}{R^{2}} \\
& +\frac{(N+n) c_{1}^{2}}{R^{2}}\left(n+N+2+\frac{n+N}{2 \sqrt{A}}\right)+\frac{(N+n) \sqrt{(N+n) K} c_{1}}{R} \\
& +\left(2+\frac{1}{\sqrt{A}}\right)(n+N) K,
\end{aligned}
$$

where $A=(N+n)(\alpha+1)(\alpha+2)$ and $c_{1}, c_{2}$ are absolute positive constants.

For the parabolic equation, Li and Yau [5] developed the fundamental gradient estimate, which is now widely called the Li-Yau estimate, for any positive solution $u(x, t)$ of the heat equation on a Riemannian manifold $M$ and showed how the classical Harnack inequality can be derived from their gradient estimate. Later, Hamiton [3] got the matrix Harnack estimate for the heat equation. Recently, Zhu [10] investigated the nonlinear parabolic equation

$$
u_{t}=\triangle u+\lambda(x, t) u^{\alpha}(x, t),
$$

where $0<\alpha<1$ and $\lambda(x, t)$ is a function defined on $M \times(-\infty, 0]$, which is $C^{1}$ in the first variable and $C^{0}$ in the second variable. The author got a Hamilton-type estimate and a Liouville-type theorem for positive solutions of (1.3).

Theorem 1.2 (Zhu [10]). Let $M$ be a Riemannian manifold of dimension $n \geq 2$ with $\operatorname{Ric}(M) \geq-k$ for some $k \geq 0$. Suppose that $u$ is a positive solution to the equation (1.3) in $Q_{R, T} \equiv B\left(x_{0}, R\right) \times\left[t_{0}-T, t_{0}\right] \subset M \times(-\infty, \infty)$. Suppose also that $u \leq \widetilde{M}$ and $\frac{|\nabla \lambda|^{2}}{\lambda} \leq \theta$ in $Q_{R, T}$. Then there exists a constant $C=C(\alpha, \widetilde{M})$ such that

$$
\frac{|\nabla u|}{u} \leq C \widetilde{M}^{1-\alpha}\left(\frac{1}{R}+\frac{1}{\sqrt{T}}+\sqrt{k}\right)+C \theta^{\frac{1}{4}} \widetilde{M}^{\frac{2}{3}(1-\alpha)}
$$

in $Q_{\frac{R}{2}, \frac{T}{2}}$.

But in the above work, the authors considered the gradient estimates for positive solutions to nonlinear equation on noncompact manifolds with fixed metric, it is natural to ask how the gradient estimates vary if the metric on 
manifolds evolves with the time. Perelman [7] showed a gradient estimate for the fundamental solution of the conjugate heat equation

$$
\triangle u-R u+\partial_{t} u=0,
$$

under Ricci flow on a closed Riemannian manifold $M$, where $R$ is the scalar curvature. Kuang and Zhang [4] established a point-wise gradient estimate for all positive solutions of the conjugate heat equation under Ricci flow. Liu [6] got first order gradient estimates for positive solutions of the heat equations on complete noncompact or closed Riemannian manifolds under Ricci flow and the author derived Harnack inequalities and second order gradient estimates for positive solutions of the heat equations under this flow. Later, Sun [8] extended these results to general geometric flow.

In this paper, we will study the interesting Li-Yau type estimate for the positive solutions of (1.1) on complete noncompact manifolds with evolving metric.

We present our main results about the equation (1.1) as follows.

Theorem 1.3. Let $(M, g(t))$ be a smooth one-parameter family of complete Riemannian manifolds evolving by

$$
\frac{\partial}{\partial t} g=2 h
$$

for $t$ in some time interval $[0, T]$. Let $M$ be complete under the initial metric $g(0)$. Given $x_{0} \in M$, and $M_{1}, R>0$, let $u$ be a positive solution to the nonlinear equation (1.1) with $u \leq M_{1}$ in the cube $Q_{2 R, T}:=\left\{(x, t) \mid d\left(x, x_{0}, t\right) \leq 2 R, 0 \leq\right.$ $t \leq T\}$. Suppose that there exist constants $K_{1}, K_{2}, K_{3}, K_{4} \geq 0$ such that

$$
\text { Ric } \geq-K_{1} g,-K_{2} g \leq h \leq K_{3} g,|\nabla h| \leq K_{4}
$$

on $Q_{2 R, T}$. Moreover, assume that $\triangle b \leq \theta$ and $|\nabla b| \leq \gamma$ in $Q_{2 R, T}$ for some positive constants $\theta$ and $\gamma$. Then for any constant $0<\beta<1$ and $(x, t) \in Q_{R, T}$, if $\beta<\sigma<1$, we have

$$
\beta \frac{|\nabla u|^{2}}{u^{2}}-b u^{\sigma-1}-\frac{u_{t}}{u} \leq H_{1}+H_{2}+\frac{n}{\beta} \frac{1}{t}
$$

where

$$
\begin{aligned}
H_{1}=\frac{n}{\beta} & \left(\frac{(n-1)\left(1+\sqrt{K_{1}} R\right) c_{1}^{2}+c_{2}+2 c_{1}^{2}}{R^{2}}+\sqrt{c_{3}} K_{2}+|b|(1-\sigma) M_{1}^{\sigma-1}\right. \\
& \left.\quad+\frac{n c_{1}^{2}}{2 \beta(1-\beta) R^{2}}\right) \\
H_{2}=[ & \frac{n^{2}}{4 \beta^{2}(1-\beta)^{2}}\left(|b|(\sigma-1)(\beta-\sigma) M_{1}^{\sigma-1}+2(\sigma-\beta) M_{1}^{\sigma-1}+2(1-\beta) K_{3}\right. \\
& \left.+2 \beta K_{1}+\frac{3}{2} K_{4}\right)+\frac{n}{\beta}\left(M_{1}^{\sigma-1} \theta+n\left(\frac{1}{\beta}\left(K_{2}+K_{3}\right)^{2}+\frac{3}{2} K_{4}\right)\right. \\
& \left.\left.+2(\sigma-\beta) M_{1}^{\sigma-1} \gamma^{2}\right)\right]^{\frac{1}{2}}
\end{aligned}
$$


and $0<\beta<1, c_{1}, c_{2}, c_{3}$ are positive constants.

Let $R \rightarrow \infty$, we can get the global Li-Yau type gradient estimates for the nonlinear parabolic equation (1.1).

Corollary 1.4. Let $(M, g(0))$ be a complete noncompact Riemannian manifold without boundary, and let $g(t)$ evolve by (1.4) for $t \in[0, T]$ and satisfy

$$
\text { Ric } \geq-K_{1} g,-K_{2} g \leq h \leq K_{3} g,|\nabla h| \leq K_{4} .
$$

Moreover, assume that $\triangle b \leq \theta(M)$ and $|\nabla b| \leq \gamma(M)$ in $M \times[0, T)$ for some constants $\theta$ and $\gamma$. Let $u$ be a positive solution of (1.1) with $u \leq M_{1}$. Then for any constant $0<\beta<1$, if $\beta<\sigma<1$, we have

$$
\beta \frac{|\nabla u|^{2}}{u^{2}}-b u^{\sigma-1}-\frac{u_{t}}{u} \leq \overline{H_{1}}+H_{2}+\frac{n}{\beta} \frac{1}{t}
$$

where

$$
\overline{H_{1}}=\sqrt{c_{3}} K_{2}+\frac{n}{\beta}|b|(1-\sigma) M_{1}^{\sigma-1},
$$

As an application, we get the following Harnack inequality.

Theorem 1.5. Let $(M, g(0))$ be a complete noncompact Riemannian manifold without boundary, and let $g(t)$ evolve by (1.4) for $t \in[0, T)$ and satisfy

$$
\text { Ric } \geq-K_{1} g,-K_{2} g \leq h \leq K_{3} g,|\nabla h| \leq K_{4} .
$$

Assume that $\triangle b \leq \theta(M)$ and $|\nabla b| \leq \gamma(M)$ in $M \times[0, T)$ for some constants $\theta$ and $\gamma$. Let $u(x, t)$ be a positive smooth solution to the equation

$$
u_{t}=\triangle u-b u^{\sigma}
$$

on $M \times[0, T)$, where $b$ is a nonpositive constant. Then if $\beta<\sigma<1$, for any points $\left(x_{1}, t_{1}\right)$ and $\left(x_{2}, t_{2}\right)$ on $M \times[0, T)$ with $0<t_{1}<t_{2}$, we have the following Harnack inequality:

$$
u\left(x_{1}, t_{1}\right) \leq u\left(x_{2}, t_{2}\right)\left(\frac{t_{2}}{t_{1}}\right)^{\frac{n}{\beta}} e^{\phi\left(x_{1}, x_{2}, t_{1}, t_{2}\right)+\overline{H_{1}}+H_{2}\left(t_{2}-t_{1}\right)},
$$

where $\phi\left(x_{1}, x_{2}, t_{1}, t_{2}\right)=\inf _{\gamma} \int_{t_{1}}^{t_{2}} \frac{1}{4 \beta}|\dot{\gamma}|^{2} d t, \overline{H_{1}}=\sqrt{c_{3}} K_{2}+\frac{n}{\beta}|b|(1-\sigma) M_{1}^{\sigma-1}$, and

$$
\begin{aligned}
H_{2}=[ & \frac{n^{2}}{4 \beta^{2}(1-\beta)^{2}}\left(|b|(\sigma-1)(\beta-\sigma) M_{1}^{\sigma-1}+2(\sigma-\beta) M_{1}^{\sigma-1}+2(1-\beta) K_{3}\right. \\
& \left.+2 \beta K_{1}+\frac{3}{2} K_{4}\right)+\frac{n}{\beta}\left(M_{1}^{\sigma-1} \theta+n\left(\frac{1}{\beta}\left(K_{2}+K_{3}\right)^{2}+\frac{3}{2} K_{4}\right)\right. \\
& \left.\left.+2(\sigma-\beta) M_{1}^{\sigma-1} \gamma^{2}\right)\right]^{\frac{1}{2}} .
\end{aligned}
$$




\section{Proof of Theorem 1.3}

Let $u$ be a positive solution to (1.1). Set $w=\ln u$, then $w$ satisfies the equation

$$
w_{t}=\Delta w+|\nabla w|^{2}-b e^{(\sigma-1) w} .
$$

In order to prove the main Theorem 1.3, we need the following lemmas.

Lemma 2.1 (Sun [8]). Suppose the metric evolves by (1.4). Then, for any smooth function $w$, we have

$$
\frac{\partial}{\partial t}|\nabla w|^{2}=-2 h(\nabla w, \nabla w)+2 \nabla w \nabla w_{t}
$$

and

$$
\frac{\partial}{\partial t} \triangle w=\triangle \frac{\partial}{\partial t} w-2 h \nabla^{2} w-2 \nabla w\left(\operatorname{div} h-\frac{1}{2} \nabla\left(t r_{g} h\right)\right),
$$

here divh is the divergence of $h$.

Lemma 2.2. Assume that $(M, g(t))$ satisfies the hypotheses of Theorem 1.3, we have

$$
\begin{aligned}
& \left(\triangle-\frac{\partial}{\partial t}\right) F \\
\geq & -2 \nabla w \nabla F+t\left\{\frac{\beta}{n}\left(|\nabla w|^{2}-b e^{(\sigma-1) w}-w_{t}\right)^{2}\right. \\
& +\left[b(\sigma-1)(\beta-\sigma) e^{(\sigma-1) w}+2(\beta-1) K_{3}-2 \beta K_{1}-\frac{3}{2} K_{4}\right]|\nabla w|^{2} \\
& \left.+2(\beta-\sigma) e^{(\sigma-1) w} \nabla w \nabla b-e^{(\sigma-1) w} \triangle b-n\left[\frac{1}{\beta}\left(K_{2}+K_{3}\right)^{2}+\frac{3}{2} K_{4}\right]\right\} \\
& +b(\sigma-1) e^{(\sigma-1) w} F-\frac{F}{t},
\end{aligned}
$$

where

$$
F=t\left(\beta|\nabla w|^{2}-b e^{(\sigma-1) w}-w_{t}\right),
$$

and $\beta$ is any constant satisfying $0<\beta<1$.

Proof. Define

By the Bochner formula, we have

$$
F=t\left(\beta|\nabla w|^{2}-b e^{(\sigma-1) w}-w_{t}\right)
$$

$$
\triangle|\nabla w|^{2} \geq 2\left|\nabla^{2} w\right|^{2}+2 \nabla w \nabla(\triangle w)-2 K_{1}|\nabla w|^{2} .
$$

Noting that

$$
\begin{aligned}
\triangle w_{t}= & (\triangle w)_{t}+2 h \nabla^{2} w+2 \nabla w\left(\operatorname{div} h-\frac{1}{2} \nabla\left(t r_{g} h\right)\right) \\
= & -\left(|\nabla w|^{2}\right)_{t}+b_{t} e^{(\sigma-1) w}+b(\sigma-1) e^{(\sigma-1) w} w_{t}+w_{t t} \\
& +2 h \nabla^{2} w+2 \nabla w\left(\operatorname{div} h-\frac{1}{2} \nabla\left(t r_{g} h\right)\right) \\
= & 2 h(\nabla w, \nabla w)-2 \nabla w \nabla w_{t}+b_{t} e^{(\sigma-1) w}+b(\sigma-1) e^{(\sigma-1) w} w_{t}+w_{t t}
\end{aligned}
$$




$$
+2 h \nabla^{2} w+2 \nabla w\left(\operatorname{div} h-\frac{1}{2} \nabla\left(\operatorname{tr}_{g} h\right)\right)
$$

and

$$
\begin{aligned}
\triangle w & =-|\nabla w|^{2}+b e^{(\sigma-1) w}+w_{t} \\
& =\left(1-\frac{1}{\beta}\right)\left(b e^{(\sigma-1) w}+w_{t}\right)-\frac{F}{\beta t} \\
& =(\beta-1)|\nabla w|^{2}-\frac{F}{t},
\end{aligned}
$$

we know,

$$
\triangle F=t\left(\beta \triangle|\nabla w|^{2}-\triangle\left(b e^{(\sigma-1) w}\right)-\triangle w_{t}\right) .
$$

By the above computation, we obtain

$$
\begin{aligned}
\beta \triangle|\nabla w|^{2} \geq & 2 \beta\left|\nabla^{2} w\right|^{2}+2 \beta \nabla w \nabla(\triangle w)-2 \beta K_{1}|\nabla w|^{2} \\
= & 2 \beta\left|\nabla^{2} w\right|^{2}+2 \beta \nabla w \nabla\left[\left(1-\frac{1}{\beta}\right)\left(b e^{(\sigma-1) w}+w_{t}\right)-\frac{F}{\beta t}\right] \\
& -2 \beta K_{1}|\nabla w|^{2} \\
= & 2 \beta\left|\nabla^{2} w\right|^{2}+2(\beta-1) e^{(\sigma-1) w} \nabla w \nabla b \\
& +2 b(\beta-1)(\sigma-1) e^{(\sigma-1) w}|\nabla w|^{2}+2(\beta-1) \nabla w \nabla w_{t} \\
& -\frac{2}{t} \nabla w \nabla F-2 \beta K_{1}|\nabla w|^{2},
\end{aligned}
$$

and

$$
\begin{aligned}
\triangle\left(b e^{(\sigma-1) w}\right)= & e^{(\sigma-1) w} \triangle b+2(\sigma-1) e^{(\sigma-1) w} \nabla w \nabla b+b(\sigma-1)^{2} e^{(\sigma-1) w}|\nabla w|^{2} \\
& +b(\sigma-1) e^{(\sigma-1) w} \triangle w \\
= & e^{(\sigma-1) w} \triangle b+2(\sigma-1) e^{(\sigma-1) w} \nabla w \nabla b+b(\sigma-1)^{2} e^{(\sigma-1) w}|\nabla w|^{2} \\
& +b(\sigma-1) e^{(\sigma-1) w}\left[(\beta-1)|\nabla w|^{2}-\frac{F}{t}\right] .
\end{aligned}
$$

So, we have

$$
\begin{aligned}
\triangle F \geq & t\left\{2 \beta\left|\nabla^{2} w\right|^{2}+2(\beta-1) e^{(\sigma-1) w} \nabla w \nabla b\right. \\
& +2 b(\beta-1)(\sigma-1) e^{(\sigma-1) w}|\nabla w|^{2}+2(\beta-1) \nabla w \nabla w_{t} \\
& -\frac{2}{t} \nabla w \nabla F-2 \beta K|\nabla w|^{2}-e^{(\sigma-1) w} \triangle b-2(\sigma-1) e^{(\sigma-1) w} \nabla w \nabla b \\
& -b(\sigma-1)^{2} e^{(\sigma-1) w}|\nabla w|^{2}-b(\sigma-1) e^{(\sigma-1) w}\left[(\beta-1)|\nabla w|^{2}-\frac{F}{t}\right] \\
& -\left(-\left(|\nabla w|^{2}\right)_{t}+2 h \nabla^{2} w+2 \nabla w\left(\operatorname{div} h-\frac{1}{2} \nabla\left(\operatorname{tr}_{g} h\right)\right)\right)+b_{t} e^{(\sigma-1) w} \\
& \left.\left.+b(\sigma-1) e^{(\sigma-1) w} w_{t}+w_{t t}\right)\right\}
\end{aligned}
$$


and

$$
\begin{aligned}
& F_{t} \\
= & \frac{F}{t}+t\left(\beta\left(|\nabla w|^{2}\right)_{t}-b_{t} e^{(\sigma-1) w}-b(\sigma-1) e^{(\sigma-1) w} w_{t}-w_{t t}\right) \\
= & \frac{F}{t}+t\left(2 \beta \nabla w \nabla w_{t}-2 \beta h(\nabla w, \nabla w)-b_{t} e^{(\sigma-1) w}-b(\sigma-1) e^{(\sigma-1) w} w_{t}-w_{t t}\right) .
\end{aligned}
$$

This implies that

$$
\begin{aligned}
& \left(\Delta-\frac{\partial}{\partial t}\right) F \\
\geq & -2 \nabla w \cdot \nabla F+t\left\{2 \beta\left|\nabla^{2} w\right|^{2}+2(\beta-1) h(\nabla w, \nabla w)\right. \\
& +b(\sigma-1)(\beta-\sigma) e^{(\sigma-1) w}|\nabla w|^{2}+2(\beta-\sigma) e^{(\sigma-1) w} \nabla w \nabla b \\
& \left.\left.-e^{(\sigma-1) w} \triangle b-2 \beta K_{1}|\nabla w|^{2}-2 h \nabla^{2} w-2 \nabla w\left(\operatorname{div} h-\frac{1}{2} \nabla\left(\operatorname{tr}_{g} h\right)\right)\right)\right\} \\
& +b(\sigma-1) e^{(\sigma-1) w} F-\frac{F}{t} .
\end{aligned}
$$

By our assumption, we have

$$
-\left(K_{2}+K_{3}\right) g \leq h \leq\left(K_{2}+K_{3}\right) g,
$$

which implies that

$$
|h|^{2} \leq\left(K_{2}+K_{3}\right)^{2}|g|^{2}=n\left(K_{2}+K_{3}\right)^{2} .
$$

Applying those bounds and Young's inequality yields

$$
\left|h \nabla^{2} w\right| \leq \frac{\beta}{2}\left|\nabla^{2} w\right|^{2}+\frac{1}{2 \beta}|h|^{2} \leq \frac{\beta}{2}\left|\nabla^{2} w\right|^{2}+\frac{n}{2 \beta}\left(K_{2}+K_{3}\right)^{2} .
$$

On the other hand,

$$
\left|\operatorname{div} h-\frac{1}{2} \nabla\left(t r_{g} h\right)\right|=\left|g^{i j} \nabla_{i} h_{j l}-\frac{1}{2} g^{i j} \nabla_{l} h_{i j}\right| \leq \frac{3}{2}|g||\nabla h| \leq \frac{3}{2} \sqrt{n} K_{4} .
$$

Finally, with the help of the inequality

$$
\left|\nabla^{2} w\right|^{2} \geq \frac{1}{n}\left(\operatorname{tr} \nabla^{2} w\right)^{2}=\frac{1}{n}(\triangle w)^{2}=\frac{1}{n}\left(|\nabla w|^{2}-b e^{(\sigma-1) w}-w_{t}\right)^{2},
$$

we get

$$
\begin{aligned}
\left(\triangle-\frac{\partial}{\partial t}\right) F \geq & -2 \nabla w \nabla F+t\left\{\frac{\beta}{n}\left(|\nabla w|^{2}-b e^{(\sigma-1) w}-w_{t}\right)^{2}\right. \\
& +b(\sigma-1)(\beta-\sigma) e^{(\sigma-1) w}|\nabla w|^{2}+2(\beta-\sigma) e^{(\sigma-1) w} \nabla w \nabla b \\
& -e^{(\sigma-1) w} \triangle b+2(\beta-1) K_{3}|\nabla w|^{2}-2 K_{1} \beta|\nabla w|^{2} \\
& \left.-\frac{n}{\beta}\left(K_{2}+K_{3}\right)^{2}-3 \sqrt{n} K_{4}|\nabla w|\right\}+b(\sigma-1) e^{(\sigma-1) w} F-\frac{F}{t} .
\end{aligned}
$$

Since

$$
3 \sqrt{n} K_{4}|\nabla w| \leq 3 K_{4}\left(\frac{n}{2}+\frac{|\nabla w|^{2}}{2}\right)
$$


we get

$$
\begin{aligned}
& \left(\triangle-\frac{\partial}{\partial t}\right) F \\
\geq & -2 \nabla w \nabla F+t\left\{\frac{\beta}{n}\left(|\nabla w|^{2}-b e^{(\sigma-1) w}-w_{t}\right)^{2}\right. \\
& +\left[b(\sigma-1)(\beta-\sigma) e^{(\sigma-1) w}+2(\beta-1) K_{3}-2 \beta K_{1}-\frac{3}{2} K_{4}\right]|\nabla w|^{2} \\
& \left.+2(\beta-\sigma) e^{(\sigma-1) w} \nabla w \nabla b-e^{(\sigma-1) w} \triangle b-n\left[\frac{1}{\beta}\left(K_{2}+K_{3}\right)^{2}+\frac{3}{2} K_{4}\right]\right\} \\
& +b(\sigma-1) e^{(\sigma-1) w} F-\frac{F}{t} .
\end{aligned}
$$

We complete the proof of Lemma 2.2.

We take a $C^{2}$ cut-off function $\tilde{\varphi}$ defined on $[0, \infty)$ such that $\tilde{\varphi}(r)=1$ for $r \in[0,1], \tilde{\varphi}(r)=0$ for $r \in[2, \infty)$, and $0 \leq \tilde{\varphi}(r) \leq 1$. Furthermore $\tilde{\varphi}$ satisfies

$$
-\frac{\tilde{\varphi}^{\prime}(r)}{\tilde{\varphi}^{\frac{1}{2}}(r)} \leq c_{1}
$$

and

$$
\tilde{\varphi}^{\prime \prime}(r) \geq-c_{2}
$$

for some absolute constants $c_{1}, c_{2}>0$. Set

$$
\varphi(x, t)=\tilde{\varphi}\left(\frac{r(x, t)}{R}\right),
$$

where $r(x, t)=d\left(x, x_{0}, t\right)$. Using an argument of Calabi [2], we can assume $\varphi(x, t) \in C^{2}(M)$ with support in $Q_{2 R, T}$. Direct calculation shows that on $Q_{2 R, T}$

$$
\frac{|\nabla \varphi|^{2}}{\varphi} \leq \frac{c_{1}^{2}}{R^{2}}
$$

By the Laplace comparison theorem in [1],

$$
\triangle \varphi \geq-\frac{(n-1)\left(1+\sqrt{K_{1}} R\right) c_{1}^{2}+c_{2}}{R^{2}} .
$$

For any $0<T_{1}<T$, let $\left(x_{0}, t_{0}\right)$ be a point in the cube $Q_{2 R, T_{1}}$, at which $\varphi F$ attains its maximum value. we can assume that this value is positive (otherwise the proof is trivial). At the point $\left(x_{0}, t_{0}\right)$, we have

$$
\nabla(\varphi F)=0, \triangle(\varphi F) \leq 0,(\varphi F)_{t} \geq 0
$$

It follows that

$$
0 \geq\left(\triangle-\frac{\partial}{\partial t}\right)(\varphi F)=(\triangle \varphi) F-\varphi_{t} F+\varphi\left(\triangle-\frac{\partial}{\partial t}\right) F+2 \nabla \varphi \nabla F
$$

By Sun [8] on p. 494, we know there exists a positive constant $c_{3}$ such that

$$
-\varphi_{t} F \geq-\sqrt{c_{3}} K_{2} F \text {. }
$$


So we obtain

$$
\varphi\left(\triangle-\frac{\partial}{\partial t}\right) F+F \triangle \varphi-\varphi_{t} F-2 F \varphi^{-1}|\nabla \varphi|^{2} \leq 0 .
$$

This inequality together with the inequalities (2.2) and (2.3) yield

$$
\varphi\left(\triangle-\frac{\partial}{\partial t}\right) F \leq H F
$$

where

$$
H=\frac{(n-1)\left(1+\sqrt{K_{1}} R\right) c_{1}^{2}+c_{2}+2 c_{1}^{2}}{R^{2}}+\sqrt{c_{3}} K_{2} .
$$

At $\left(x_{0}, t_{0}\right)$, by Lemma 2.2 and $(2.4)$, we have

$$
\begin{aligned}
& 0 \geq \varphi\left(\triangle-\frac{\partial}{\partial t}\right) F-H F \geq-H F+\varphi\left\{-\frac{F}{t_{0}}+b(\sigma-1) e^{(\sigma-1) w} F\right. \\
& +\frac{\beta t_{0}}{n}\left(|\nabla w|^{2}-b e^{(\sigma-1) w}-w_{t}\right)^{2}-2 \nabla w \cdot \nabla F \\
& +t_{0}\left[b(\sigma-1)(\beta-\sigma) e^{(\sigma-1) w}+2(\beta-1) K_{3}-2 \beta K_{1}-\frac{3}{2} K_{4}\right]|\nabla w|^{2} \\
& \left.+2 t_{0}(\beta-\sigma) e^{(\sigma-1) w} \nabla w \nabla b-t_{0} e^{(\sigma-1) w} \triangle b-n t_{0}\left[\frac{1}{\beta}\left(K_{2}+K_{3}\right)^{2}+\frac{3}{2} K_{4}\right]\right\} \\
& \geq-H F-\varphi t_{0}^{-1} F+2 F \nabla w \nabla \varphi+\frac{\beta t_{0}}{n} \varphi\left(|\nabla w|^{2}-b e^{(\sigma-1) w}-w_{t}\right)^{2} \\
& +b(\sigma-1) e^{(\sigma-1) w} \varphi F+t_{0} \varphi\left[b(\sigma-1)(\beta-\sigma) e^{(\sigma-1) w}+2(\beta-1) K_{3}\right. \\
& \left.-2 \beta K_{1}-\frac{3}{2} K_{4}\right]|\nabla w|^{2}+2 t_{0} \varphi(\beta-\sigma) e^{(\sigma-1) w} \nabla w \nabla b-t_{0} \varphi e^{(\sigma-1) w} \triangle b \\
& -n t_{0} \varphi\left[\frac{1}{\beta}\left(K_{2}+K_{3}\right)^{2}+\frac{3}{2} K_{4}\right] \\
& \geq-H F-\varphi t_{0}^{-1} F+2 F \nabla w \nabla \varphi+\frac{\beta t_{0}}{n} \varphi\left(|\nabla w|^{2}-b e^{(\sigma-1) w}-w_{t}\right)^{2} \\
& +|b|(\sigma-1) M_{1}^{\sigma-1} \varphi F-t_{0} \varphi\left[|b|(\sigma-1)(\beta-\sigma) M_{1}^{\sigma-1}+2(1-\beta) K_{3}\right. \\
& \left.+2 \beta K_{1}+\frac{3}{2} K_{4}\right]|\nabla w|^{2}-t_{0} \varphi M_{1}^{\sigma-1} \theta-n t_{0} \varphi\left[\frac{1}{\beta}\left(K_{2}+K_{3}\right)^{2}+\frac{3}{2} K_{4}\right] \\
& +2 t_{0} \varphi(\beta-\sigma) M_{1}^{\sigma-1}|\nabla w| \gamma \\
& \geq-H F-\varphi t_{0}^{-1} F+2 F \nabla w \nabla \varphi+\frac{\beta t_{0}}{n} \varphi\left(|\nabla w|^{2}-b e^{(\sigma-1) w}-w_{t}\right)^{2} \\
& +|b|(\sigma-1) M_{1}^{\sigma-1} \varphi F-t_{0} \varphi\left[|b|(\sigma-1)(\beta-\sigma) M_{1}^{\sigma-1}+2(\sigma-\beta) M_{1}^{\sigma-1}\right. \\
& \left.+2(1-\beta) K_{3}+2 \beta K_{1}+\frac{3}{2} K_{4}\right]|\nabla w|^{2}-t_{0} \varphi\left[M_{1}^{\sigma-1} \theta\right. \\
& \left.+n\left(\frac{1}{\beta}\left(K_{2}+K_{3}\right)^{2}+\frac{3}{2} K_{4}\right)+2(\sigma-\beta) M_{1}^{\sigma-1} \gamma^{2}\right] \text {. }
\end{aligned}
$$


Set

$\widetilde{C_{1}}=|b|(\sigma-1)(\beta-\sigma) M_{1}^{\sigma-1}+2(\sigma-\beta) M_{1}^{\sigma-1}+2(1-\beta) K_{3}+2 \beta K_{1}+\frac{3}{2} K_{4}$,

and

$$
\widetilde{C_{2}}=M_{1}^{\sigma-1} \theta+n\left(\frac{1}{\beta}\left(K_{2}+K_{3}\right)^{2}+\frac{3}{2} K_{4}\right)+2(\sigma-\beta) M_{1}^{\sigma-1} \gamma^{2} .
$$

Multiplying both sides of the above inequality by $t_{0} \varphi$, noting the fact that $0<\varphi<1$, we have

$$
\begin{aligned}
0 \geq & -H t_{0} \varphi F-\varphi F+2 t_{0} \varphi F \nabla w \nabla \varphi+|b|(\sigma-1) M_{1}^{\sigma-1} t_{0} \varphi F \\
& +\frac{\beta t_{0}^{2}}{n} \varphi^{2}\left(|\nabla w|^{2}-b e^{(\sigma-1) w}-w_{t}\right)^{2}-\widetilde{C_{1}} t_{0}^{2} \varphi^{2}|\nabla w|^{2}-\widetilde{C_{2}} t_{0}^{2} \varphi^{2} \\
\geq & -H t_{0} \varphi F-\varphi F-\frac{2 c_{1}}{R} t_{0} \varphi F|\nabla w| \varphi^{\frac{3}{2}}+|b|(\sigma-1) M_{1}^{\sigma-1} t_{0} \varphi F \\
& +\frac{\beta t_{0}^{2}}{n} \varphi^{2}\left[\left(|\nabla w|^{2}-b e^{(\sigma-1) w}-w_{t}\right)^{2}-\frac{n}{\beta} \widetilde{C_{1}}|\nabla w|^{2}\right]-\widetilde{C_{2}} t_{0}^{2} \varphi^{2}
\end{aligned}
$$

where the last inequality used

$$
-2 \varphi \nabla w \nabla F=2 F \nabla w \nabla \varphi \geq-2 F|\nabla w||\nabla \varphi| \geq-\frac{2 c_{1}}{R} \varphi^{\frac{1}{2}} F|\nabla w| .
$$

Let

It follows that

$$
y=\varphi|\nabla w|^{2}, z=\varphi\left(b e^{(\sigma-1) w}+w_{t}\right)
$$

$$
\begin{aligned}
0 \geq & \varphi F\left(-H t_{0}+|b|(\sigma-1) M_{1}^{\sigma-1} t_{0}-1\right)-\frac{2 c_{1}}{R} t_{0} F|\nabla w| \varphi^{\frac{3}{2}} \\
& +\frac{\beta t_{0}^{2}}{n} \varphi^{2}\left[|\nabla w|^{2}-\frac{n}{\beta} \widetilde{C_{1}}|\nabla w|^{2}\right]-\widetilde{C_{2}} t_{0}^{2} \varphi^{2} \\
\geq & \varphi F\left(-H t_{0}+|b|(\sigma-1) M_{1}^{\sigma-1} t_{0}-1\right) \\
& +\frac{\beta t_{0}^{2}}{n}\left\{(y-z)^{2}-\frac{n}{\beta} \widetilde{C_{1}} y-2 n c_{1} R^{-1} y^{\frac{1}{2}}\left(y-\frac{1}{\beta} z\right)\right\}-\widetilde{C_{2}} t_{0}^{2} .
\end{aligned}
$$

Using the inequality $a x^{2}-b x \geq-\frac{b^{2}}{4 a}$, valid for $a, b>0$, one obtains

$$
\begin{aligned}
& \frac{\beta t_{0}^{2}}{n}\left\{(y-z)^{2}-\frac{n}{\beta} \widetilde{C_{1}} y-2 \frac{n c_{1}}{R} y^{\frac{1}{2}}\left(y-\frac{z}{\beta}\right)\right\} \\
= & \frac{\beta t_{0}^{2}}{n}\left\{\beta^{2}\left(y-\frac{z}{\beta}\right)^{2}+(1-\beta)^{2} y^{2}-\frac{n}{\beta} \widetilde{C_{1}} y+\left(2\left(\beta-\beta^{2}\right) y-\frac{n c_{1}}{R} y^{\frac{1}{2}}\right)\left(y-\frac{z}{\beta}\right)\right\} \\
\geq & \frac{\beta t_{0}^{2}}{n}\left\{\beta^{2}\left(y-\frac{z}{\beta}\right)^{2}-\frac{n^{2}{\widetilde{C_{1}}}^{2}}{4 \beta^{2}(1-\beta)^{2}}-\frac{n^{2} c_{1}^{2}}{2 R^{2}\left(\beta-\beta^{2}\right)}\left(y-\frac{z}{\beta}\right)\right\} \\
= & \frac{\beta}{n}(\varphi F)^{2}-\frac{n{\widetilde{C_{1}}}^{2} t_{0}^{2}}{4 \beta(1-\beta)^{2}}-\frac{n c_{1}^{2} t_{0}}{2 R^{2}\left(\beta-\beta^{2}\right)}(\varphi F) .
\end{aligned}
$$


Hence,

$$
\begin{aligned}
& \frac{\beta}{n}(\varphi F)^{2}+\left[-H t_{0}+|b|(\sigma-1) M_{1}^{\sigma-1} t_{0}-1-\frac{n c_{1}^{2} t_{0}}{2 R^{2}\left(\beta-\beta^{2}\right)}\right](\varphi F) \\
& -\frac{n{\widetilde{C_{1}}}^{2} t_{0}^{2}}{4 \beta(1-\beta)^{2}}-{\widetilde{C_{2}}}_{2} t_{0}^{2} \leq 0 .
\end{aligned}
$$

From the inequality $A x^{2}-2 B x \leq C$, we have $x \leq \frac{2 B}{A}+\sqrt{\frac{C}{A}}$.

We can get

$$
\begin{aligned}
\varphi F \leq & \frac{n}{\beta}\left(H t_{0}+|b|(1-\sigma) M_{1}^{\sigma-1} t_{0}+1+\frac{n c_{1}^{2} t_{0}}{2 R^{2}\left(\beta-\beta^{2}\right)}\right) \\
& +\left[\frac{n}{\beta}\left(\frac{n \widetilde{C}_{1}^{2}}{4 \beta(1-\beta)^{2}}+\widetilde{C_{2}}\right)\right]^{\frac{1}{2}} t_{0} .
\end{aligned}
$$

If $d\left(x, x_{0}, T_{1}\right)<R$, we have $\varphi\left(x, T_{1}\right)=1$. Then

$$
\begin{aligned}
F\left(x, T_{1}\right)= & T_{1}\left(\beta|\nabla w|^{2}+c e^{-w(\alpha+1)}-w_{t}\right) \\
\leq & \varphi F\left(x_{0}, t_{0}\right) \\
\leq & \frac{n}{\beta}\left(H t_{0}+|b|(1-\sigma) M_{1}^{\sigma-1} t_{0}+1+\frac{n c_{1}^{2} t_{0}}{2 R^{2}\left(\beta-\beta^{2}\right)}\right) \\
& +\left[\frac{n}{\beta}\left(\frac{n \widetilde{C}_{1}^{2}}{4 \beta(1-\beta)^{2}}+\widetilde{C_{2}}\right)\right]^{\frac{1}{2}} t_{0} .
\end{aligned}
$$

As $T_{1}$ is arbitrary, we can get the case (1) of Theorem 1.3.

Proof of Theorem 1.5. For any points $\left(x_{1}, t_{1}\right)$ and $\left(x_{2}, t_{2}\right)$ on $M \times[0, T)$ with $0<t_{1}<t_{2}$, we take a curve $\gamma(t)$ parameterized with $\gamma\left(t_{1}\right)=x_{1}$ and $\gamma\left(t_{2}\right)=x_{2}$. One gets from Corollary 1.4 that

$$
\begin{aligned}
& \log u\left(x_{2}, t_{2}\right)-\log u\left(x_{1}, t_{1}\right) \\
= & \int_{t_{1}}^{t_{2}}\left((\log u)_{t}+\langle\nabla \log u, \dot{\gamma}\rangle\right) d t \\
\geq & \int_{t_{1}}^{t_{2}}\left(\beta|\nabla \log u|^{2}-\frac{n}{\beta t}-b u^{\sigma-1}-\overline{H_{1}}-H_{2}-|\nabla \log u \| \dot{\gamma}|\right) d t \\
\geq & -\int_{t_{1}}^{t_{2}}\left(\frac{1}{4 \beta}|\dot{\gamma}|^{2}+\frac{n}{\beta t}+b u^{\sigma-1}+\overline{H_{1}}+H_{2}\right) d t \\
= & -\left(\int_{t_{1}}^{t_{2}} \frac{1}{4 \beta}|\dot{\gamma}|^{2} d t+\log \left(\frac{t_{2}}{t_{1}}\right)^{\frac{n}{\beta}}+\overline{H_{1}}+H_{2}\left(t_{2}-t_{1}\right)\right),
\end{aligned}
$$

which means that

$$
\log \frac{u\left(x_{1}, t_{1}\right)}{u\left(x_{2}, t_{2}\right)} \leq \int_{t_{1}}^{t_{2}} \frac{1}{4 \beta}|\dot{\gamma}|^{2} d t+\log \left(\frac{t_{2}}{t_{1}}\right)^{\frac{n}{\beta}}+\overline{H_{1}}+H_{2}\left(t_{2}-t_{1}\right) .
$$


Therefore,

$$
u\left(x_{1}, t_{1}\right) \leq u\left(x_{2}, t_{2}\right)\left(\frac{t_{2}}{t_{1}}\right)^{\frac{n}{\beta}} e^{\phi\left(x_{1}, x_{2}, t_{1}, t_{2}\right)+\overline{H_{1}}+H_{2}\left(t_{2}-t_{1}\right)},
$$

where $\phi\left(x_{1}, x_{2}, t_{1}, t_{2}\right)=\inf _{\gamma} \int_{t_{1}}^{t_{2}} \frac{1}{4 \beta}|\dot{\gamma}|^{2} d t, \overline{H_{1}}=\sqrt{c_{3}} K_{2}+\frac{n}{\beta}|b|(1-\sigma) M_{1}^{\sigma-1}$, and

$$
\begin{aligned}
H_{2}=[ & \frac{n^{2}}{4 \beta^{2}(1-\beta)^{2}}\left(|b|(\sigma-1)(\beta-\sigma) M_{1}^{\sigma-1}+2(\sigma-\beta) M_{1}^{\sigma-1}+2(1-\beta) K_{3}\right. \\
& \left.+2 \beta K_{1}+\frac{3}{2} K_{4}\right)+\frac{n}{\beta}\left(M_{1}^{\sigma-1} \theta+n\left(\frac{1}{\beta}\left(K_{2}+K_{3}\right)^{2}+\frac{3}{2} K_{4}\right)\right. \\
& \left.\left.+2(\sigma-\beta) M_{1}^{\sigma-1} \gamma^{2}\right)\right]^{\frac{1}{2}}
\end{aligned}
$$

Acknowledgement. The authors would like to thank the editor and the anonymous referees for their valuable comments and helpful suggestions that improved the quality of the paper. Moreover, the author would like to thank his supervisor Professor Kefeng Liu for his constant encouragement and help. This work is supported by Postdoctoral Science Foundation of China (2013M531342) and the Fundamental Research Funds for the Central Universities (NS2012065).

\section{References}

[1] T. Aubin, Nonlinear Analysis on Manifolds. Monge-Ampré equations, Springer, New York, 1982.

[2] E. Calabi, An extension of E. Hopf's maximum principle with an application to Riemannian geometry, Duke Math. J. 25 (1958), no. 1, 45-56.

[3] R. Hamilton, A matrix Harnack estimate for the heat equation, Comm. Anal. Geom. 1 (1993), no. 1, 113-126.

[4] S. Kuang and Q. S. Zhang, A gradient estimate for all positive solutions of the conjugate heat equation under Ricci flow, J. Funct. Anal. 255 (2008), no. 4, 1008-1023.

[5] P. Li and S. T. Yau, On the parabolic kernel of the Schrödinger operator, Acta Math. 156 (1986), no. 3-4, 153-201.

[6] S. P. Liu, Gradient estimates for solutions of the heat equation under Ricci flow, Pacific J. Math. 243 (2009), no. 1, 165-180.

[7] G. Perelman, The entropy formula for the Ricci flow and its geometric applications, preprint, (2002), arXiv math.DG/0211159.

[8] J. Sun, Gradient estimates for positive solutions of the heat equation under geometric flow, Pacific J. Math. 253 (2011), no. 2, 489-510.

[9] J. Zhang and B. Q. Ma, Gradient estimates for a nonlinear equation $\triangle_{f} u+c u^{-\alpha}=0$ on complete noncompact manifolds, Commun. Math. 19 (2011), no. 1, 73-84.

[10] X. B. Zhu, Gradient estimates and Liouville theorems for nonlinear parabolic equations on noncompact Riemannian manifolds, Nonlinear Anal. 74 (2011), no. 15, 5141-5146.

Department of Mathematics

Nanjing University of Aeronautics and Astronautics

NANJiNG 210016, P. R. ChINA

E-mail address: zhaozongliang09@163.com 